\title{
Erratum to: Advanced Data Mining and Applications
}

\author{
Jinyan $\mathrm{Li}^{1(\bowtie)}$, Xue $\mathrm{Li}^{2}$, Shuliang Wang ${ }^{3}$, Jianxin $\mathrm{Li}^{4}$, \\ and Quan Z. Sheng ${ }^{5}$ \\ ${ }^{1}$ University of Technology Sydney, Ultimo, NSW, Australia \\ jinyan.li@uts.edu.au \\ ${ }^{2}$ University of Queensland, Brisbane, QLD, Australia \\ ${ }^{3}$ Beijing Institute of Technology, Beijing, China \\ ${ }^{4}$ University of Western Australia, Crawley, WA, Australia \\ ${ }^{5}$ University of Adelaide, Adelaide, SA, Australia
}

\section{Erratum to:}

\section{Chapter 52}

\section{Partitioning Clustering Based on Support Vector Ranking DOI: 10.1007/978-3-319-49586-6_52}

The original version of the paper starting on p. 726 was revised. An acknowledgement has been added. The original chapter was corrected.

\section{Erratum to:}

\section{Chapter 59}

\section{Sentiment Analysis for Depression Detection on Social Networks DOI: 10.1007/978-3-319-49586-6_59}

The original version of the paper starting on p. 807 was revised. An acknowledgement has been added. The original chapter was corrected.

The updated original online version for this chapters can be found at 